\title{
Multiple-Decision Procedures for Testing the Homogeneity of Mean for $k$ Exponential Distributions
}

\author{
Han-Ching Chen, Her Pei Shan, and Nae-Sheng Wang \\ Department of Statistics, Feng Chia University, No. 100, Wenhua Road, Xitun District, Taichung City 407, Taiwan \\ Correspondence should be addressed to Han-Ching Chen; hang.ching11@msa.hinet.net
}

Received 25 June 2014; Accepted 4 August 2014; Published 19 August 2014

Academic Editor: Yunqiang Yin

Copyright (C) 2014 Han-Ching Chen et al. This is an open access article distributed under the Creative Commons Attribution License, which permits unrestricted use, distribution, and reproduction in any medium, provided the original work is properly cited.

In multiple-decision procedures, a crucial objective is to determine the association between the probability of a correct decision (CD) and the sample size. A review of some methods is provided, including a subset selection formulation proposed by Huang and Panchapakesan, a multidecision procedure for testing the homogeneity of means by Huang and Lin, and a similar procedure for testing the homogeneity of variances by Lin and Huang. In this paper, we focus on the use of the Lin and Huang method for testing the null hypothesis $H_{0}$ of homogeneity of means for $k$ exponential distributions. We discuss the decision rule $R$, evaluation of the critical value $C$, and the infimum of $P(C D \mid R)$ for $k$ independent random samples from $k$ exponential distributions. In addition, we also observed that a lower bound for the probability of CD relative to the number of the common sample size is determined based on the desired probability of CD when the largest mean is sufficiently larger than the other means. We explain the results by using two examples.

\section{Introduction}

A multiple-decision problem can be defined as a situation where a person or a group of people must select the number of possible actions from a given finite set. Gupta and Huang [1] and Lin and Gupta [2] presented the selection procedures relevant to multiple-decision theory, including indifference zone selection and subset selection. They suggested that preferences among alternatives can be determined by maximizing the expected value of a numerical utility function or equivalently minimizing the expected value of a loss function. They indicated that the subset selection procedures have been studied and applied widely in determining the required sample size, which is the number of replications or batches used for selecting the optimal population among $k$ populations and for selecting a subset.

Huang and Panchapakesan [3] suggested a modification of the subset selection formulation on the largest mean and the smallest variance. Huang and Lin [4] presented a multidecision procedure for testing the homogeneity of means when the sample sizes and unknown variance are unequal. Lin and Huang [5] used a similar procedure for testing the hypothesis $H_{0}$ regarding the homogeneity of the variances. The purpose of this paper was to use the Lin and Huang method for testing the hypothesis $H_{0}$ regarding the homogeneity of the means for $k$ exponential distributions. When $H_{0}$, the hypothesis, is rejected, the main objective was to obtain a nonempty subset $E$ of the $k$ populations that will include the population related to the largest means (called the best population). In this case, a correct decision (CD) is said to occur if the selected subset $E$ contains the best populations.

The paper is organized as follows. In Section 2, we introduce the definitions and notations of decision rule $R$ for $k$ exponential distributions. In Section 3, we discuss the evaluation of the critical value of our test and the infimum of the probability of a correct decision CD. In Section 4, the performance of the method is illustrated with two examples and the behavior of our procedure is analyzed. Finally, concluding remarks are provided in Section 5.

\section{Related Concepts of the Decision Rule}

In this section, we use the Lin and Huang [5] method to identify the decision rule $R$ for $k$ exponential distributions. 
Let $X_{i 1}, X_{i 2}, \ldots, X_{i n_{i}}, i=1,2, \ldots, k$, be $k$ independent random samples from $k$ exponential distribution $\Gamma\left(1, \theta_{i}\right), \theta_{i}>$ $0, i=1,2, \ldots, k$. We define the $\tau_{i}=\theta_{i} /\left(\prod_{j=1}^{k} \theta_{j}\right)^{1 / k}$ as the distance between $\theta_{i}$ and all other $\theta_{j}^{\prime}$ s.

Then the MLE of $\tau_{i}$ is

$$
\widehat{\tau}_{i}=\frac{\widehat{\theta}_{i}}{\left(\prod_{j=1}^{k} \widehat{\theta}_{j}\right)^{1 / k}},
$$

where

$$
\widehat{\theta}_{i}=\frac{1}{n_{i}} \sum_{j=1}^{n_{i}} X_{i j}=\bar{X}_{i .} \quad i=1,2, \ldots, k .
$$

For testing $H_{0}: \theta_{1}=\theta_{2}=\ldots, \theta_{k}$, the test statistic that arises naturally is $\max _{1 \leq i \leq k} \widehat{\tau}_{i}$.

We now present the steps of decision rule $R$ for $k$ exponential distribution as follows.

First, given $\alpha$, where $0<\alpha<1$, we want to find a $C$ such that the condition

$$
P\left\{\max _{1 \leq i \leq k} \widehat{\tau}_{i} \geq C \mid H_{0}\right\} \leq \alpha,
$$

where $C$ is the critical value for the decision rule $R$ and $\alpha$ is a given probability of Type I error at level $\alpha$.

Second, given $\Delta>0$ and $P^{*}$, where $1 / k<P^{*}<1$, we want to find a nonempty subset $E=\left\{1 \leq j \leq k \mid \widehat{\tau}_{j} \geq C\right\}$ of the $k$ populations that contains the best populations and it is necessary that $\inf _{\underline{\theta} \in \Omega_{\Delta}} P\{\mathrm{CD} \mid R\} \geq P^{*}$, where $P(\mathrm{CD} \mid$ $R)=P_{\Omega_{\Delta}}\left(\max _{1 \leq i \leq k} \widehat{\tau}_{i} \geq C\right.$ and $\left.\widehat{\tau}_{(k)} \geq C\right)$ and $\Omega_{\Delta}=\{\underline{\theta}=$ $\left.\left(\theta_{1}, \ldots, \theta_{k}\right) \mid \tau_{[k]} \geq \Delta\right\}$, where $\tau_{[1]} \leq \tau_{[2]} \leq \cdots \leq \tau_{[k]}$ denote the ordered $\tau_{i}$ and $\widehat{\tau}_{i}=\widehat{\theta}_{i} /\left(\prod_{j=1}^{k} \widehat{\theta}_{j}\right)^{1 / k}$ and $\widehat{\theta}_{(k)}$ is associated with the population having the largest $\theta_{[k]}$.

\section{Assessment of the Critical Value $C$ and the Infimum of $P(\mathrm{CD} \mid R)$}

In this section, we want to estimate the critical value $C$ and the infimum of $P(\mathrm{CD} \mid R)$ for $k$ exponential distribution.

Lemma 1. Let $X_{i 2}, \ldots, X_{i n_{i}}, i=1,2, \ldots, k$, be $k$ independent random samples from $k$ exponential distribution $\Gamma\left(1, \theta_{i}\right), i=$ $1,2, \ldots, k$. The MLE of $\theta_{i}$ is

$$
\begin{gathered}
\widehat{\theta}_{i}=\frac{1}{n_{i}} \sum_{j=1}^{n_{i}} X_{i j}=\bar{X}_{i .} \quad i=1,2, \ldots, k, \\
n_{i} \widehat{\theta}_{i} \sim \Gamma\left(n_{i}, \theta i\right), \quad \frac{\widehat{\theta}_{i}}{\hat{\theta}_{i}} \sim \Gamma\left(n_{i}, \frac{1}{n_{i}}\right) .
\end{gathered}
$$

Lemma 2. The MLE of $\tau_{i}$ is

$$
\begin{gathered}
\widehat{\tau}_{i}=\frac{\widehat{\theta}_{i}}{\left(\prod_{j=1}^{k} \widehat{\theta}_{j}\right)^{1 / k}}, \quad \text { for } \widehat{\theta}_{i}=\bar{X}_{i .} i=1,2, \ldots, k, \\
\ln \widehat{\tau}_{i}-\ln \tau_{i}=\sum_{j=1}^{k} c_{j} \frac{\ln \widehat{\theta}_{j}}{\theta_{j}}
\end{gathered}
$$

where

$$
c_{j}= \begin{cases}1-\frac{1}{k} & j=i, \\ -\frac{1}{k} & j \neq i .\end{cases}
$$

Thus, $\ln \widehat{\tau}_{i}-\ln \tau_{i}$ is a linear combination of independent $\log$ gamma random variables with coefficients $(1-(1 / k))$ for $\ln \left(\widehat{\theta}_{i} / \theta_{i}\right)$ and $-1 / k$ for $\ln \left(\widehat{\theta}_{j} / \theta_{j}\right), j=1,2, \ldots, k$.

Lemma 3. According to the Lin and Huang [5] appendix, we can get

$$
\operatorname{Pr}\left(\ln \widehat{\tau}_{i}-\ln \tau_{i}<C\right) \cong \frac{1}{1+\exp \left\{-\pi\left(C-b_{i}\right) / \sqrt{3 a_{i}}\right\}},
$$

where

$$
a_{i}=\frac{(k-1)^{2}}{k^{2} n_{i}}+\frac{1}{k^{2}} \sum_{\substack{j=1 \\ j \neq i}}^{k} \frac{1}{n_{j}}, \quad b_{i}=-\frac{1}{2 n_{i}}+\frac{1}{k} \sum_{j=1}^{k} \frac{1}{2 n_{j}} .
$$

Theorem 4. Under the same assumption of Lemma 1, for testing $H_{0}: \theta_{1}=\theta_{2}=\ldots, \theta_{k}$, given the samples sizes $n_{1}$, $\ldots, n_{k}$ and $0<\alpha<1$, the critical value $C$ for the decision rule $R$ satisfies the $\operatorname{Pr}\left\{\max _{1 \leq i \leq k} \widehat{\tau}_{i} \geq C \mid H_{0}\right\} \leq \alpha$ which is approximately $C=\exp \left(\left(\sqrt{3 a_{[k]}} / \pi\right) \ln ((k / \alpha)-1)+b_{[k]}\right)$, where $a_{[k]}=\max _{i} a_{i}, b_{[k]}=\max _{i} b_{i}$ and $\left(a_{i}, b_{i}\right)$ are given by (8). Further, given $\Delta>0$, one then has $\inf _{\theta \in \Omega_{\Delta}} P\{\mathrm{CD} \mid R\} \geq$ $1 /\left(1+\exp \left\{\pi\left(\ln C-\Delta-b_{[1]}\right) / \sqrt{3 a_{[1]}}\right\}\right)=P^{*}$, where $b_{[1]}=\min _{i} b_{i}$ and $a_{[1]}=\min _{i} a_{i}$.

Proof. Under $H_{0}$, we have $\tau_{i}=\theta_{i} /\left(\prod_{j=1}^{k} \theta_{j}\right)^{1 / k}=1$ for each $i=1,2, \ldots, k$.

Therefore, the $\ln \tau_{i}=0$ for each $i=1,2, \ldots, k$. And

$$
\begin{aligned}
\operatorname{Pr} & \left\{\max _{1 \leq i \leq k} \widehat{\tau}_{i} \geq C \mid H_{0}\right\} \\
& =\operatorname{Pr}\left\{\max _{1 \leq i \leq k} \ln \widehat{\tau}_{i} \geq \ln C, \text { for some } i=1, \ldots, k \mid H_{0}\right\} \\
& =\operatorname{Pr}\left\{\ln \widehat{\tau}_{i}-\ln \tau_{i} \geq \ln C-\ln \tau_{i}, \text { for some } i,\right. \\
& \left.\quad i=1,2, \ldots, k \mid H_{0}\right\} \\
& \leq \sum_{i=1}^{k} \operatorname{Pr}\left\{\ln \widehat{\tau}_{i}-\ln \tau_{i} \geq \ln C-\ln \tau_{i}\right\} \\
& =\sum_{i=1}^{k} \operatorname{Pr}\left\{\ln \widehat{\tau}_{i}-\ln \tau_{i} \geq \ln C\right\} \quad\left(\operatorname{since} \text { the } \ln \tau_{i}=0\right) \\
& \cong \sum_{i=1}^{k}\left(1-\frac{1}{1+\exp \left\{-\pi\left(\ln C-b_{i}\right) / \sqrt{3 a_{i}}\right\}}\right)
\end{aligned}
$$

(by Lemma 3)

$$
\begin{aligned}
& =\sum_{i=1}^{k}\left[\frac{1}{1+\exp \left\{\left(\pi \ln C-b_{i}\right) / \sqrt{3 a_{i}}\right\}}\right] \\
& \leq \frac{k}{1+\exp \left\{\pi\left(\ln C-b_{[k]}\right) / \sqrt{3 a_{[k]}}\right\}} \equiv \alpha \quad \text { (say). }
\end{aligned}
$$


TABLE 1: Times to breakdown (in minutes) at each of the five voltage levels.

\begin{tabular}{lcc}
\hline Voltage level $(\mathrm{kV})$ & $n_{1}$ & Breakdown times \\
\hline 30 & 11 & $17.05,22.66,21.02,175.88,139.07,144.12,20.46,43.40,194.90,47.30,7.74$ \\
32 & 15 & $0.40,82.85,9.88,89.29,215.10,2.75,0.79,15.93,3.91,0.27,0.69,100.58,27.80,13.95,53.24$ \\
34 & 19 & $0.96,4.15,0.19,0.78,8.01,31.75,7.35,6.50,8.27,33.91,32.52,3.16,4.85,2.78,4.67,1.31,12.06,36.71,72.89$ \\
36 & 15 & $1.97,0.59,2.58,1.69,2.71,25.50,0.35,0.99,3.99,3.67,2.07,0.96,5.35,2.90,13.77$ \\
38 & 8 & $0.47,0.73,1.40,0.74,0.39,1.13,0.09,2.38$ \\
\hline
\end{tabular}

TABLE 2: Computed values.

\begin{tabular}{cccccc}
\hline & 1 & 2 & 3 & 4 & 5 \\
\hline$n_{i}$ & 11 & 15 & 19 & 15 & 8 \\
$a_{i}$ & 0.0706 & 0.0561 & 0.0477 & 0.0561 & 0.0911 \\
$b_{i}$ & -0.0053 & 0.0069 & 0.0139 & 0.0069 & -0.0223 \\
$\widehat{\theta}_{i}$ & 75.981 & 41.174 & 14.35895 & 4.606 & 0.9162 \\
$\widehat{\tau}_{i}$ & 6.6858 & 3.623 & 1.2635 & 0.4053 & 0.0806 \\
\hline
\end{tabular}

We have

$$
\begin{gathered}
1+\exp \left\{\frac{\pi\left(\ln C-b_{[k]}\right)}{\sqrt{3 a_{[k]}}}\right\}=\frac{k}{\alpha}, \\
\ln C-b_{[k]}=\frac{\sqrt{3} \sqrt{a_{[k]}}}{\pi} \ln \left(\frac{k}{\alpha}-1\right) .
\end{gathered}
$$

Therefore, the critical value $C$ is

$$
C=\exp \left(\frac{\sqrt{3 a_{[k]}}}{\pi} \ln \left(\frac{k}{\alpha}-1\right)+b_{[k]}\right)
$$

However

$$
\begin{aligned}
\inf _{\underline{\theta} \in \Omega_{\Delta}} P\{C D \mid R\} \\
\quad=\inf _{\underline{\theta} \in \Omega_{\Delta}}\left\{\operatorname{Pr}\left(\widehat{\tau}_{(k)} \geq C\right)\right\} \\
\quad=\inf _{\underline{\theta} \in \Omega_{\Delta}}\left\{\operatorname{Pr}\left(\ln \widehat{\tau}_{(k)} \geq \ln c\right)\right\} \\
\geq \inf _{\underline{\theta} \in \Omega_{d}} p\left(\ln \widehat{\tau}_{(k)}-\ln \tau_{[k]} \geq \ln C-\ln \Delta\right) \\
\simeq 1-\frac{1}{1+\exp \left\{-\pi\left(\ln C-\ln \Delta-b_{(k)}\right) / \sqrt{3 a_{(k)}}\right\}} \\
\quad=\frac{1}{1+\exp \left\{\pi\left(\ln C-\ln \Delta-b_{(k)}\right) / \sqrt{3 a_{(k)}}\right\}} \\
\quad \geq \frac{1}{1+\exp \left\{\pi\left(\ln C-\ln \Delta-b_{[1]}\right) / \sqrt{3 a_{[1]}}\right\}} \equiv P^{*},
\end{aligned}
$$

which is the desired result.
Theorem 5. Under the same assumption of Lemma 1 and assuming $n_{1}=n_{2}=\cdots=n_{k}=n$, given level $\alpha$, where $0<\alpha<1$, and $n$, the critical value $C$ is

$$
C=\exp \left(\frac{\sqrt{3}}{\pi} \sqrt{\frac{(k-1)}{k n}} \ln \left(\frac{k}{\alpha}-1\right)\right) .
$$

Furthermore, given $P^{*}$, where $1 / k<P^{*}<1$ and $\Delta>0$, under the decision rule that $R$ satisfies $\operatorname{Pr}\left\{\max _{1 \leq i \leq k} \ln \widehat{\tau}_{i} \geq \ln C\right.$ । $\left.H_{0}\right\} \leq \alpha$ and $\inf _{\theta \in \Omega_{\Delta}} P\{\mathrm{CD} \mid R\} \geq P^{*}$, we have the common sample size $n$ as follows:

$$
n=\left[\frac{(k-1)}{k}\left(\frac{\ln \left\{(k-\alpha) P^{*}\right\}-\ln \left\{\alpha\left(1-P^{*}\right)\right\}}{(\sqrt{3} / \pi) \ln \Delta}\right)^{2}\right]+1
$$

where $[x]$ denotes the lowest integer greater than or equal to $x$. Proof. By Theorem 4, $n_{i}=n, i=1,2, \ldots, k$, we have

$$
a_{i}=\frac{k-1}{n k}, \quad b_{i}=0 \quad \text { for each } i=1,2, \ldots, k
$$

and the critical value $C$ is

$$
\begin{aligned}
C & =\exp \left(\frac{\sqrt{3 a_{(k)}}}{\pi} \ln \left(\frac{k}{\alpha}-1\right)+b_{(k)}\right) \\
& =\exp \left(\frac{\sqrt{3}}{\pi} \sqrt{\frac{k-1}{n k}} \ln \left(\frac{k}{\alpha}-1\right)+b_{(k)}\right) \\
& =\exp \left(\frac{\sqrt{3}}{\pi} \sqrt{\frac{k-1}{n k}} \ln \left(\frac{k}{\alpha}-1\right)\right)
\end{aligned}
$$

which is the desired result.

Given $P^{*}$, where $1 / k<P^{*}<1$ and $\Delta>0$, using the propriety of Theorem 4 , we have

$$
\begin{aligned}
& \inf _{\underline{\theta} \in \Omega_{\Delta}} P\{\mathrm{CD} \mid R\} \\
& \quad \geq \frac{1}{1+\exp \{\pi(\ln C-\ln \Delta) / \sqrt{3 a}\}} \stackrel{\text { Let }}{\geq} P^{*},
\end{aligned}
$$


TABLE 3: Infimum of $P\{C D \mid R\}$.

\begin{tabular}{lccccccc}
\hline$\Delta$ & 2 & 2.2 & 2.4 & 2.6 & 2.8 & 3.0 & 3.2 \\
\hline$P^{*}$ & 0.3201 & 0.5095 & 0.6815 & 0.8062 & 0.8850 & 0.9318 & 0.9589 \\
\hline
\end{tabular}

TABLE 4: Sample size $n$ for $k=3,4,5,6, \alpha=0.01(0.05)$, and $P^{*}=0.60$.

\begin{tabular}{|c|c|c|c|c|c|c|c|c|c|c|c|}
\hline \multirow{2}{*}{$k$} & \multicolumn{11}{|c|}{$\Delta$} \\
\hline & 1.5 & 1.6 & 1.7 & 1.8 & 1.9 & 2.0 & 2.1 & 2.2 & 2.3 & 2.4 & 2.5 \\
\hline 3 & $69(38)$ & $52(28)$ & $41(23)$ & 33 (19) & $28(16)$ & 24 (14) & $22(12)$ & 19 (11) & $17(10)$ & $16(9)$ & $15(9)$ \\
\hline 4 & $84(48)$ & $63(36)$ & $50(28)$ & $41(23)$ & $35(20)$ & 30 (17) & $26(15)$ & $23(14)$ & $21(12)$ & 19 (11) & $18(11)$ \\
\hline 5 & $96(56)$ & $72(42)$ & $57(33)$ & $47(27)$ & $39(23)$ & $34(20)$ & $30(18)$ & 27 (16) & $24(14)$ & $22(13)$ & $20(12)$ \\
\hline 6 & $106(62)$ & $79(47)$ & $62(37)$ & $51(30)$ & $43(26)$ & $37(22)$ & $33(20)$ & $29(18)$ & $26(16)$ & $24(14)$ & $22(13)$ \\
\hline
\end{tabular}

Note: the numbers in parentheses represent the fitted values at the level; $\alpha=0.05$.

TABle 5: Sample size $n$ for $k=3,4,5,6, \alpha=0.01(0.05)$, and $P^{*}=0.80$.

\begin{tabular}{|c|c|c|c|c|c|c|c|c|c|c|c|}
\hline \multirow{2}{*}{$k$} & \multicolumn{11}{|c|}{$\Delta$} \\
\hline & 1.5 & 1.6 & 1.7 & 1.8 & 1.9 & 2.0 & 2.1 & 2.2 & 2.3 & 2.4 & 2.5 \\
\hline 3 & $92(55)$ & $69(42)$ & $54(33)$ & $45(27)$ & $38(23)$ & $32(20)$ & $29(18)$ & $25(16)$ & $23(14)$ & $21(13)$ & $19(12)$ \\
\hline 4 & $112(69)$ & $84(51)$ & $66(41)$ & $54(33)$ & $46(28)$ & $39(24)$ & $34(22)$ & $31(19)$ & $28(17)$ & $25(16)$ & $23(15)$ \\
\hline 5 & $126(79)$ & $94(59)$ & $74(47)$ & $61(38)$ & $51(32)$ & $44(28)$ & $39(25)$ & $35(22)$ & $31(20)$ & $28(18)$ & $26(17)$ \\
\hline 6 & 138 (87) & $103(65)$ & $81(52)$ & $66(42)$ & $56(36)$ & $48(31)$ & $42(26)$ & $38(24)$ & $34(22)$ & $31(20)$ & $28(18)$ \\
\hline
\end{tabular}

Note: the numbers in parentheses represent the fitted values at the level; $\alpha=0.05$.

Using (15) and $C=\exp ((\sqrt{3} a / \pi) \sqrt{(k-1) / n k} \ln ((k / \alpha)-$ $1)$ ), we then have the minimal sample size $n$ as follows:

$$
n \geq\left[\frac{(k-1)}{k}\left(\frac{\ln \left\{(k-\alpha) P^{*}\right\}-\ln \left\{\alpha\left(1-P^{*}\right)\right\}}{(\sqrt{3} / \pi) \ln \Delta}\right)^{2}\right]+1
$$

Remark 6. The $\hat{\theta}_{i}$ defined in this study fulfills Lawless Corollary 4.1.1. (Type II censored test property) [6]. When the observations are Type II censored data, we can take $\widehat{\theta}_{i}=$ $2 T_{i} / \theta_{i}$, where $T=\sum_{i=1}^{r} t_{(i)}+(n-r) t_{(r)}$ and $t_{(r)}$ are the first $r$ ordered observation of a random sample of size $n$ from the exponential distribution. In this case, the $T=\sum_{i=1}^{r} w_{i}$, where $w_{i}=n t_{(i)}, i=1,2 \ldots, r$, remain unchanged.

\section{Examples}

In this section, we provide two examples to explain the results of performing Theorems 4 and 5 .

Example 1. This example is from Nelson [7]. In this example, the results of a life test experiment are described in which specimens of electrical insulating fluid were subjected to a constant voltage stress. The length of time until each specimen failed, or "broke down," was observed. Table 1 gives results for five groups of specimens, tested at voltages ranging from 30 to 38 kilovolts $(\mathrm{kV})$. We use the data on times to breakdown (in minutes) at each of the five voltage levels for our example.

The computed values are given in Table 2 based on the assumption that $\alpha=0.01$.

We obtained $C=2.8511$. Because $\widehat{\tau}_{1} \geq C$ and $\widehat{\tau}_{2} \geq C$, using the decision rule $R$, we reject $H_{0}: \widehat{\tau}_{1}=\widehat{\tau}_{2}=\widehat{\tau}_{3}=\widehat{\tau}_{4}=$ $\widehat{\tau}_{5}$ and select the subset containing populations 1 and 2 . We identified these two populations as contributing substantially. We claim that the select subset contains the population with the largest mean.

For selected values of $\Delta, \Delta=2(0.2) 3.2, \inf _{\underline{\theta} \in \Omega_{\Delta}} P\{\mathrm{CD}$ | $R\}=P^{*}$ is tabulated in Table 3 . The probability of a correct decision is at least 0.3201 when $\Delta$ is 2 . This probability increases to 0.9589 when $\Delta$ is 3.2.

Example 2. Based on the same assumption as Theorem 5, given the number of populations $k, k=3,4,5$, and 6 , as well as $\alpha=0.05$ and 0.01 and $\Delta=1.5(0.1) 2.5$ and $P^{*}=$ $0.6,0.8,0.9$, and 0.95 , we can determine $n$ by using (14), so that $\inf _{\underline{\theta} \in \Omega_{\Delta}} P\{\mathrm{CD} \mid R\} \geq P^{*}$. Several selected combinations of $n$ in each case are tabulated in Tables 4, 5, 6, and 7 which show the populations $k$ that have the minimal sample size $n$ required to satisfy the $P^{*}$.

\section{Concluding Remarks}

In this study, we considered the methods of the Lin and Huang theorems to propose a framework for analyzing and synthesizing multiple-decision procedures used for testing the homogeneity of means for $k$ exponential distributions [5]. 
TABle 6: Sample size $n$ for $k=3,4,5,6, \alpha=0.01(0.05)$, and $P^{*}=0.90$.

\begin{tabular}{|c|c|c|c|c|c|c|c|c|c|c|c|}
\hline \multirow{2}{*}{$k$} & \multicolumn{11}{|c|}{$\Delta$} \\
\hline & 1.5 & 1.6 & 1.7 & 1.8 & 1.9 & 2.0 & 2.1 & 2.2 & 2.3 & 2.4 & 2.5 \\
\hline 3 & $114(72)$ & $85(54)$ & $67(43)$ & $55(35)$ & $46(30)$ & $40(26)$ & $35(23)$ & $31(20)$ & $28(18)$ & $26(17)$ & $24(15)$ \\
\hline 4 & 137 (89) & $103(67)$ & $81(53)$ & $66(43)$ & $56(36)$ & $48(31)$ & $42(28)$ & $37(25)$ & $34(22)$ & $31(20)$ & 28 (19) \\
\hline 5 & 154 (101) & $115(76)$ & $91(60)$ & $74(49)$ & $63(41)$ & $54(36)$ & $47(31)$ & $42(28)$ & $38(25)$ & $34(23)$ & $31(21)$ \\
\hline 6 & 168 (111) & $125(83)$ & $99(66)$ & $81(54)$ & $68(45)$ & $58(39)$ & $51(34)$ & $45(30)$ & $41(27)$ & $37(25)$ & $34(23)$ \\
\hline
\end{tabular}

Note: the numbers in parentheses represent the fitted values at the level; $\alpha=0.05$.

TABle 7: Sample size $n$ for $k=3,4,5,6, \alpha=0.01(0.05)$, and $P^{*}=0.95$.

\begin{tabular}{|c|c|c|c|c|c|c|c|c|c|c|c|}
\hline \multirow{2}{*}{$k$} & \multicolumn{11}{|c|}{$\Delta$} \\
\hline & 1.5 & 1.6 & 1.7 & 1.8 & 1.9 & 2.0 & 2.1 & 2.2 & 2.3 & 2.4 & 2.5 \\
\hline 3 & $136(90)$ & $102(68)$ & $80(53)$ & $66(44)$ & $55(37)$ & $48(32)$ & $42(28)$ & $37(25)$ & $33(23)$ & $30(21)$ & 28 (19) \\
\hline 4 & $163(110)$ & $122(82)$ & $96(65)$ & $78(53)$ & $66(45)$ & $57(39)$ & $50(34)$ & $44(30)$ & $40(27)$ & $36(25)$ & $33(23)$ \\
\hline 5 & 183 (124) & $136(93)$ & $107(73)$ & $88(60)$ & $74(51)$ & $64(44)$ & $56(38)$ & $49(34)$ & $44(31)$ & $40(28)$ & $37(26)$ \\
\hline 6 & $198(136)$ & $148(102)$ & $116(80)$ & $95(65)$ & $80(55)$ & $69(47)$ & $60(42)$ & $53(37)$ & $48(33)$ & $44(30)$ & $40(28)$ \\
\hline
\end{tabular}

Note: the numbers in parentheses represent the fitted values at the level; $\alpha=0.05$.

We provided two examples and present the main results to explain Theorems 4 and 5 which can select the subset containing the population with the largest mean and effectively determine common sample size $n$ to satisfy the requirement of $P^{*}$. This paper presents the use of one technique to both select the optimal system among $k$ systems and construct an optimal rule for selecting a subset of $k$ independent random samples. We suggest employing the methods to facilitate the development of traditional statistical analyses used in the methodologies, techniques, and software applied in performing multiple-decision procedures for testing the homogeneity of means for $k$ exponential distributions problems, such as life testing and reliability engineering.

\section{Conflict of Interests}

The authors declare that there is no conflict of interests regarding the publication of this paper.

\section{References}

[1] S. S. Gupta and D. Y. Huang, "A note on optimal subset selection procedures," The Annals of Statistics, vol. 8, no. 5, pp. 1164-1167, 1980.

[2] X. Lin and S. S. Gupta, Multiple decision theory: Ranking and selection problems [Ph.D. thesis], Purdue University, 1999.

[3] D. Y. Huang and S. Panchapakesan, "A modified subset selection formulation with special reference to one-way and two-way layout experiments," Communications in Statistics A: Theory and Methods, vol. 5, no. 7, pp. 621-633, 1976.

[4] D. Huang and C. Lin, "Multiple decision procedures for testing homogeneity of normal means with unequal unknown variances," in Advances in Statistical Decision Theory and Applications, S. Panchapakesan and N. Balakrishnan, Eds., Statistics for Industry and Technology, pp. 253-262, Birkhäuser, Boston, Mass, USA, 1997.
[5] C. Lin and D. Huang, "On some multiple decision procedures for normal variances," Communications in Statistics: Simulation and Computation, vol. 36, no. 1-3, pp. 265-275, 2007.

[6] J. F. Lawless, Statistical Models and Methods for Lifetime Data, New York, Wiley-Interscience, 2nd edition, 2003.

[7] W. B. Nelson, "Graphical analysis of accelerated life test data with the inverse power law model," IEEE Transactions on Reliability, vol. 21, pp. 2-11, 1972. 


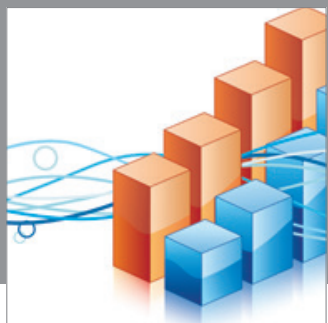

Advances in

Operations Research

mansans

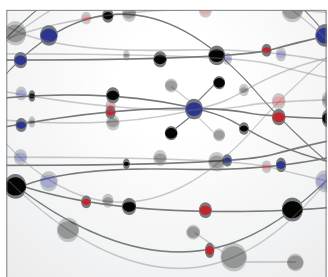

The Scientific World Journal
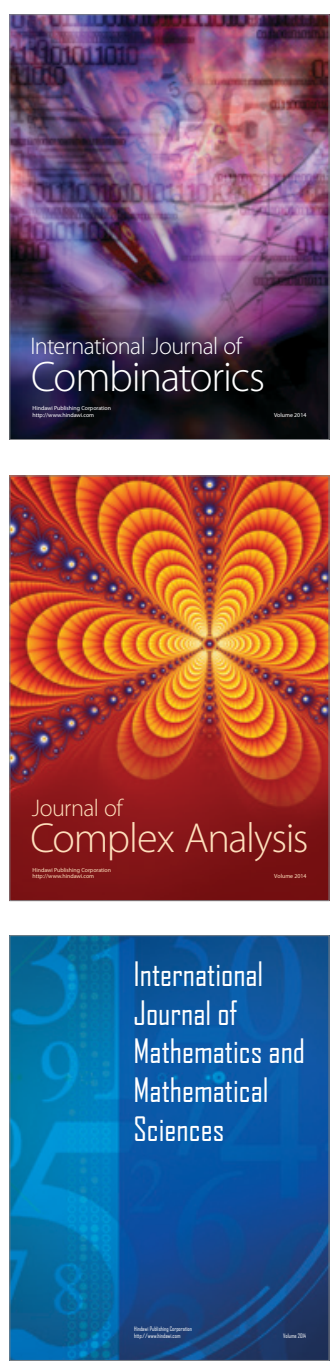
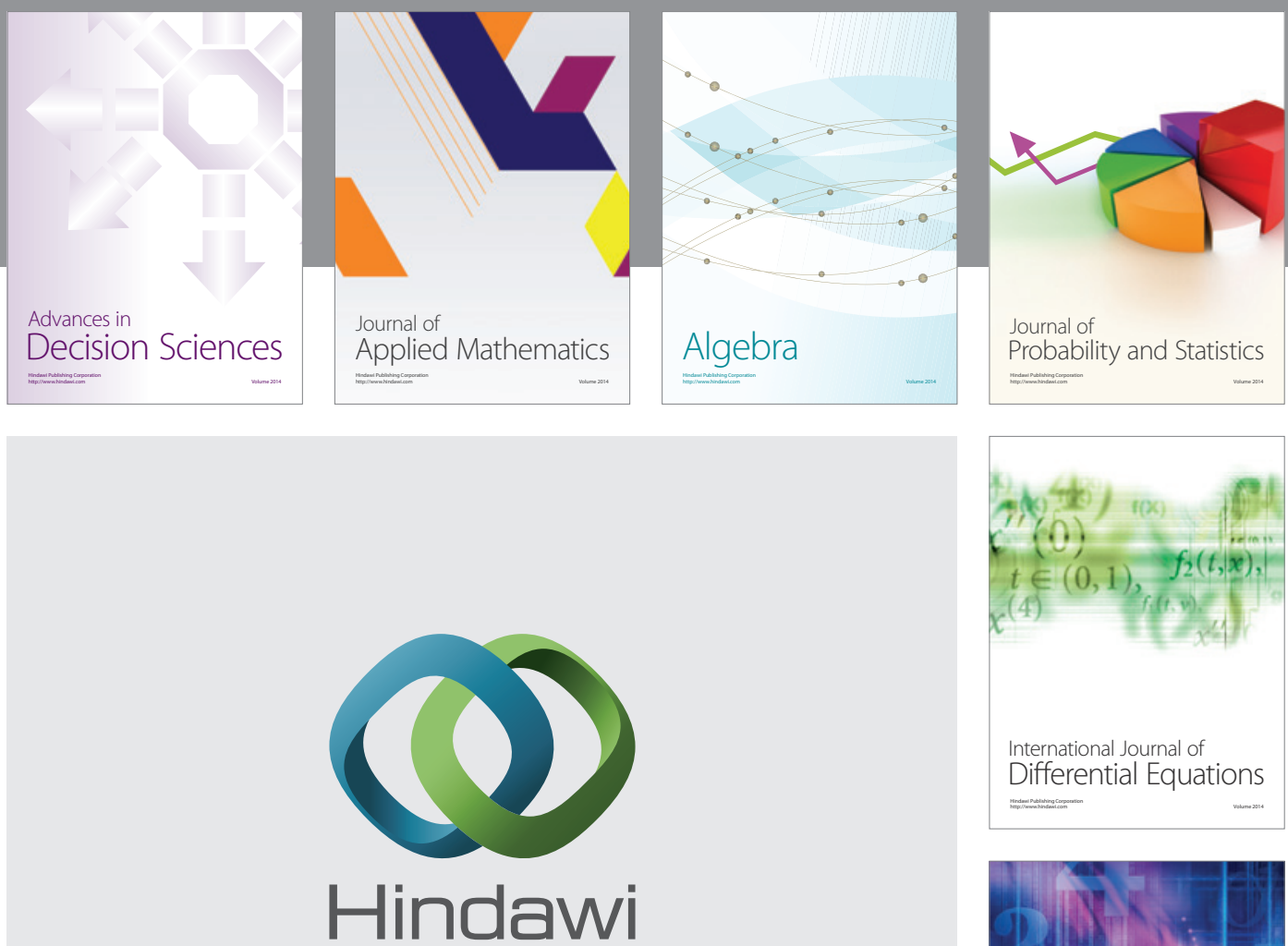

Submit your manuscripts at http://www.hindawi.com
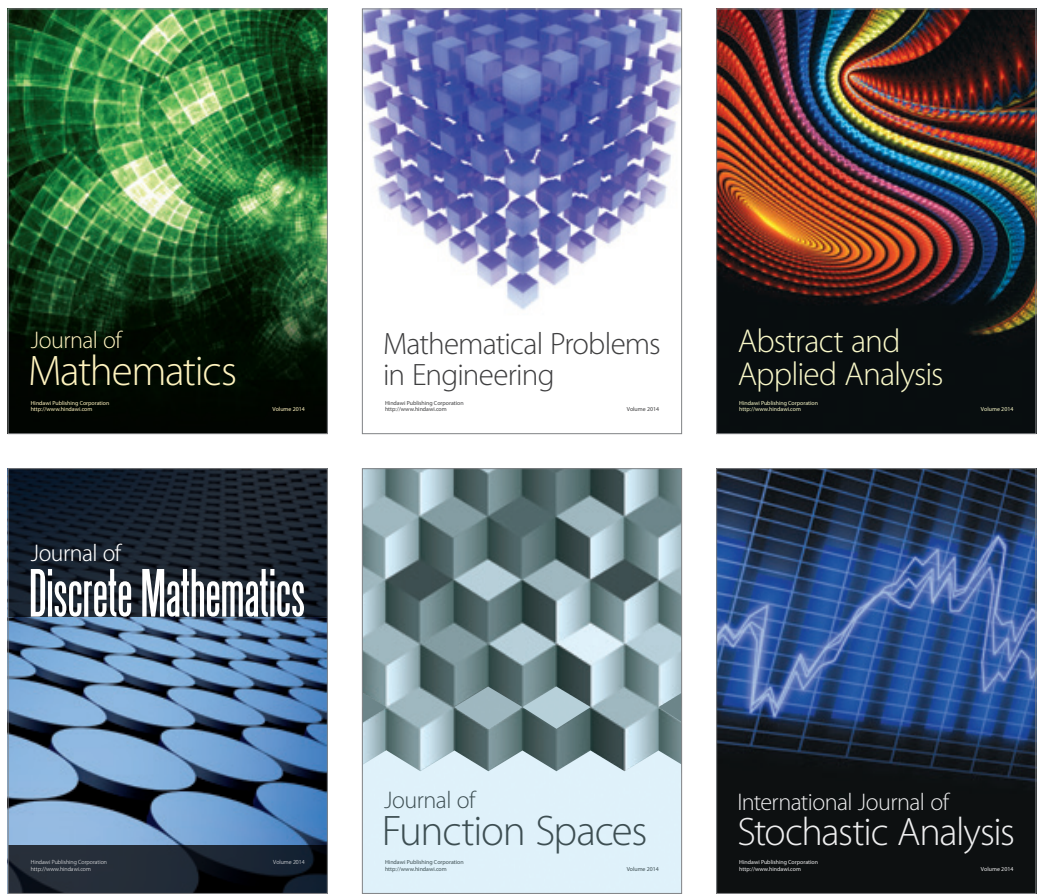

Journal of

Function Spaces

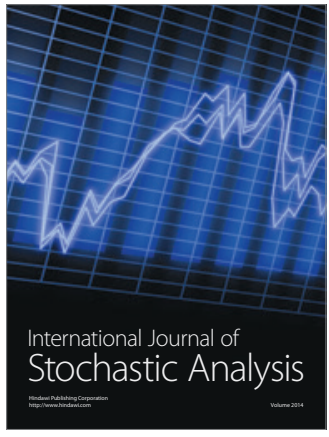

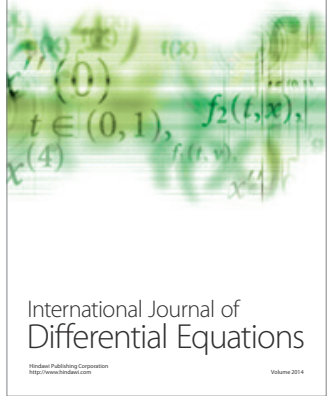
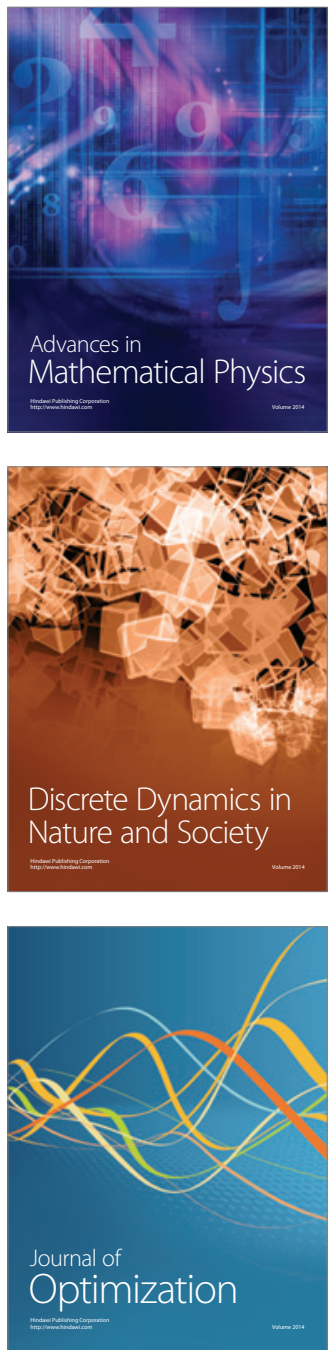